\title{
Transient Analysis of Single-Line-to-Ground Faults in Three-Phase Circuits Using Clarke Transformation
}

\author{
Diego Bellan \\ Department of Electronics, Information and Bioengineering \\ Politecnico di Milano \\ Milan, Italy \\ diego.bellan@polimi.it
}

\begin{abstract}
This work deals with the analytical solution of transients in single-line-to-ground faults in three-phase power systems through the Clarke transformation. The proposed approach is based on the Thevenin representation of the alpha, beta, and zero component circuits in the Laplace domain. The fault results in a mutual coupling of such circuits. For the singleline-to-ground fault the circuit coupling can be represented by an ideal transformer connected between the alpha and the zero component circuits. The proposed systematic methodology, however, can be readily extended to other kinds of faults. The analytical solution derived in the paper is validated through Matlab simulations in the time domain.
\end{abstract}

Index Terms - Clarke transformation, Power system analysis, Time domain analysis, Transient analysis.

\section{INTRODUCTION}

Transients analysis in electrical power systems is a key issue in power system analysis, especially when fault analysis and the related effects on system components are concerned [1]. The conventional approach foresees the use of commercial software like the ElectroMagnetic Transients Program (EMTP) for numerical simulation of the system [1]-[2]. It is well-known, however, as a general principle, that analytical solutions would provide deeper insight into theoretical and physical understanding of the system under analysis. Moreover, in many cases a system can be effectively represented through a simplified equivalent circuit for which analytical techniques could be well suited instead of the rough numerical approach. Two main problems, however, need consideration when analytical solutions come into play. First, conventional circuit analysis of three-phase systems is performed through a frequency-domain tool such as the symmetrical components transformation which is based on the assumption of circuit symmetry between the three phases [3]-[5]. Such assumption, however, is not met under fault conditions where typically only one or two phases are involved. A second point is that a transient analysis is more properly performed in the time domain instead of frequency domain. Such point can be faced by means of a time-domain tool such as the Clarke transformation traditionally used in the analysis of rotating electrical machines and power electronics [6]-[8]. To the Author's knowledge, power system analysis by means of the Clarke transformation has received very little attention in the related technical literature [2], [6]. In this paper, it is shown that the Clarke transformation is a tool very well-suited for transient analysis of three-phase circuits since it operates in the time domain, and since it results in simple coupling between alpha, beta, and zero component circuits (i.e., the modal circuits of the transformation) in case of asymmetrical faults such as the single-line-to-ground fault. The equivalent circuits derived in the paper can be readily solved through the Laplace transform. The proposed methodology is based on a rigorous and systematic circuit representation of each three-phase component and connection, thus the proposed approach can be easily extended to other fault conditions.

\section{THE CLARKE TRANSFORMATION}

The Clarke transformation operates on three phase variables (i.e., currents or voltages) in the time domain to obtain the transformed variables alpha, beta, and zero, according to:

$$
\left[\begin{array}{l}
i_{\alpha} \\
i_{\beta} \\
i_{0}
\end{array}\right]=\sqrt{\frac{2}{3}}\left[\begin{array}{ccc}
1 & -1 / 2 & -1 / 2 \\
0 & \sqrt{3} / 2 & -\sqrt{3} / 2 \\
1 / \sqrt{2} & 1 / \sqrt{2} & 1 / \sqrt{2}
\end{array}\right]\left[\begin{array}{l}
i_{a} \\
i_{b} \\
i_{c}
\end{array}\right]
$$

Notice that (1) is the so-called rational form of the Clarke transformation since the orthogonality of the transformation matrix results in the conservation of power from the $(a, b, c)$ to the $(\alpha, \beta, 0)$ variables. This property is fundamental in the derivation of equivalent circuits in the transformed domain. Moreover, notice that (1) holds also when linear operators are applied to the time domain variables, e.g., phasors under sinusoidal steady state and Laplace transform for general timedomain behavior.

In view of the transient analysis described in Section III, it is important to stress that the phasor analysis under sinusoidal conditions is needed to solve the three-phase system in the steady state prior the fault in order to evaluate the initial conditions for the fault event. In fact, before the fault event, the 
system is assumed symmetrical and under sinusoidal steady state. Therefore, from the standard Clarke analysis, we obtain three uncoupled circuits whose phasor solutions can be readily derived. Such solutions allow the calculation of the initial condition corresponding to the fault time instant. The initial conditions evaluated through the phasor analysis valid in the steady state prior the fault event will be used for a proper application of the Laplace transform to analyze the transient after the fault event.

\section{CIRCUIT REPRESENTATION OF A SINGLE-LINE-TO-GROUND FAULT}

Let us consider the reference three-phase circuit shown in Fig. 1 where the left side represents a simplified model for the source and the line, whereas the right side represents the load. The single-line-to-ground fault is taken into account by a threephase switch where only the switch between line $a$ and ground can be operated, whereas the remaining switches $b$ and $c$ hold the open-circuit state. Prior the fault event, the circuit is working under sinusoidal steady state conditions. Phasors can be used to evaluate the currents in the alpha, beta, and zero component circuits obtained through the Clarke transformation. From the phasor solutions the line currents at $t$ $=0$ (i.e., the fault time instant) can be readily evaluated in order to establish the initial conditions for the inductors. The alpha, beta, and zero component circuits in the sinusoidal steady state prior the fault event are shown in Fig. 2. Notice that to obtain the modal circuits in Fig. 2 the following two results concerning the four-terminal star connection were used. First, in alpha and beta component circuits each star centre is shorted. Second, in the zero component circuit the impedances connected to the star fourth terminal can be reported to the three-phase side by a simple multiplication by 3 . Such properties can be rigorously proved similarly to the case of the symmetrical component transformation [3].

Once the initial conditions for the alpha, beta, and zero component circuits have been evaluated, the fault takes place, i.e., the switch $a$ in Fig. 1 is operated. The constraints set by the three-phase switch on the $a, b, c$ variables:

$$
v_{a}=0, i_{b}=i_{c}=0
$$

result in constraints on the alpha, beta, zero variables according to the Clarke transformation:

$$
\begin{aligned}
& {\left[\begin{array}{l}
v_{\alpha} \\
v_{\beta} \\
v_{0}
\end{array}\right]=\sqrt{\frac{2}{3}}\left[\begin{array}{ccc}
1 & -1 / 2 & -1 / 2 \\
0 & \sqrt{3} / 2 & -\sqrt{3} / 2 \\
1 / \sqrt{2} & 1 / \sqrt{2} & 1 / \sqrt{2}
\end{array}\right]\left[\begin{array}{c}
0 \\
v_{b} \\
v_{c}
\end{array}\right]=} \\
& =\sqrt{\frac{2}{3}}\left[\begin{array}{l}
-1 / 2\left(v_{b}+v_{c}\right) \\
\sqrt{3} / 2\left(v_{b}-v_{c}\right) \\
1 / \sqrt{2}\left(v_{b}+v_{c}\right)
\end{array}\right] \\
& {\left[\begin{array}{l}
i_{\alpha} \\
i_{\beta} \\
i_{0}
\end{array}\right]=\sqrt{\frac{2}{3}}\left[\begin{array}{ccc}
1 & -1 / 2 & -1 / 2 \\
0 & \sqrt{3} / 2 & -\sqrt{3} / 2 \\
1 / \sqrt{2} & 1 / \sqrt{2} & 1 / \sqrt{2}
\end{array}\right]\left[\begin{array}{c}
i_{a} \\
0 \\
0
\end{array}\right]=\sqrt{\frac{2}{3}}\left[\begin{array}{c}
i_{a} \\
0 \\
i_{a} / \sqrt{2}
\end{array}\right]}
\end{aligned}
$$

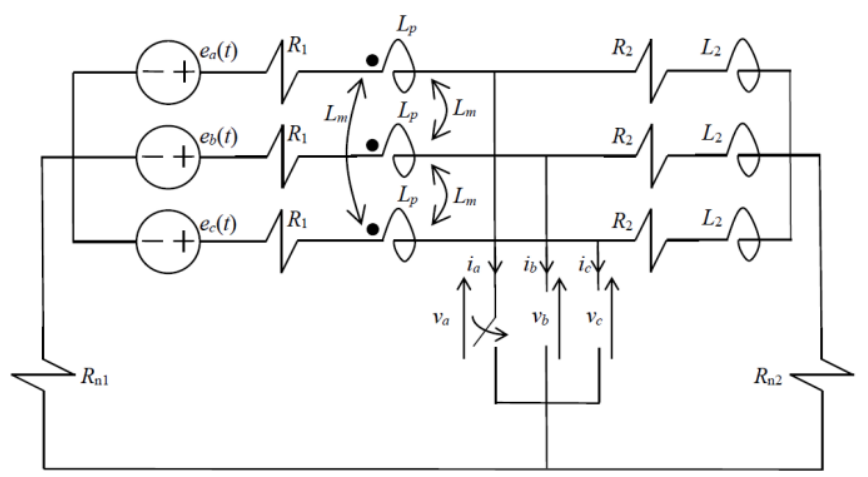

Figure 1. Reference three-phase circuit used to explain the proposed methodology.

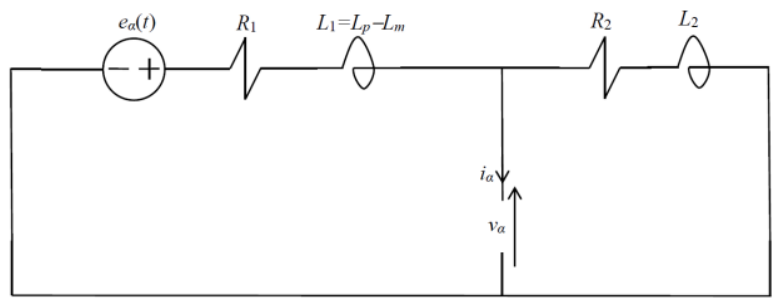

(a)

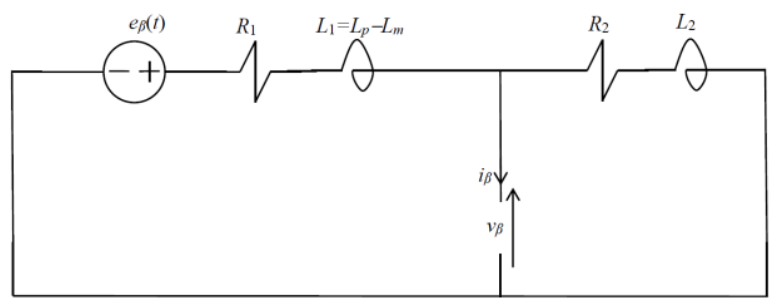

(b)

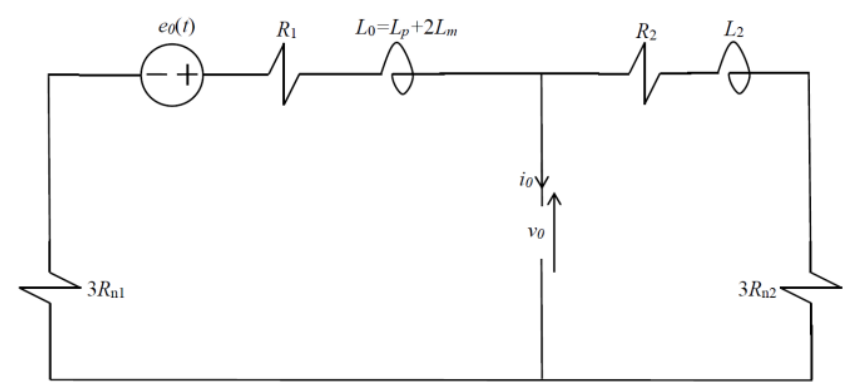

(c)

Figure 2. Alpha (a), beta (b), and zero (c) component circuits for $t<0$, i.e., prior the fault event. They can be analyzed in the phasor domain in order to calculate the inductors currents at $t=0$, i.e., the initial conditions for the subsequent transient analysis.

Thus, from (3a)-(3b) we obtain the following constraints on the Clarke variables:

$$
v_{0}=-\sqrt{2} v_{\alpha}, \quad i_{\alpha}=\sqrt{2} i_{0}, \quad i_{\beta}=0
$$

The first two equations in (4) define an ideal transformer with ratio $k=-\sqrt{2}$ between alpha and zero component circuits, whereas the third equation states that the beta 
component circuit is not involved in the transient. Therefore, the transient analysis can be performed in the Laplace domain by representing the coupling between alpha and zero component circuits through the ideal transformer defined above. The equivalent circuit is represented in Fig. 3, where the Thevenin equivalents of the alpha and zero component circuits shown in Fig. 2 are considered. Simple circuit analysis of Fig. 3 provides:

$$
I_{\alpha}(s)=\frac{V_{T \alpha}(s)-V_{T 0}(s) / k}{Z_{T \alpha}(s)+Z_{T 0}(s) / k^{2}}=\frac{V_{T \alpha}(s)+V_{T 0}(s) / \sqrt{2}}{Z_{T \alpha}(s)+Z_{T 0}(s) / 2}
$$

Finally, from (3b) we obtain the Laplace transform of the fault current $I_{a}(s)=\sqrt{3 / 2} I_{\alpha}(s)$.

For a complete analysis of the transient, the voltages $v_{\mathrm{b}}$ and $v_{\mathrm{c}}$ must be also evaluated. From the inverse Clarke transformation of (3a) we obtain:

$$
\begin{aligned}
{\left[\begin{array}{c}
0 \\
v_{b} \\
v_{c}
\end{array}\right]=} & \sqrt{\frac{2}{3}}\left[\begin{array}{ccc}
1 & 0 & 1 / \sqrt{2} \\
-1 / 2 & \sqrt{3} / 2 & 1 / \sqrt{2} \\
-1 / 2 & -\sqrt{3} / 2 & 1 / \sqrt{2}
\end{array}\right]\left[\begin{array}{l}
v_{\alpha} \\
v_{\beta} \\
v_{0}
\end{array}\right]= \\
= & \sqrt{\frac{2}{3}}\left[\begin{array}{cc}
-3 / 2 & v_{\alpha}+\sqrt{3} / 2 v_{\beta} \\
-3 / 2 & v_{\alpha}-\sqrt{3} / 2 v_{\beta}
\end{array}\right]
\end{aligned}
$$

In the Laplace domain, from Fig. 3 we have:

$$
V_{\alpha}(s)=V_{T \alpha}(s)-Z_{T \alpha}(s) I_{\alpha}(s)
$$

where $I_{\alpha}(s)$ is given by (5). The beta voltage in (6) (Laplace domain) is given by the Thevenin voltage $V_{T \beta}(s)$ of the beta component circuit not involved in the transient (i.e., open circuited at the fault location). Therefore, from (6) we obtain:

$$
\begin{aligned}
& V_{b}(s)=-\sqrt{\frac{3}{2}} V_{\alpha}(s)+\frac{1}{\sqrt{2}} V_{\beta}(s) \\
& V_{c}(s)=-\sqrt{\frac{3}{2}} V_{\alpha}(s)-\frac{1}{\sqrt{2}} V_{\beta}(s)
\end{aligned}
$$

\section{NUMERICAL VALIDATION}

The three-phase circuit shown in Fig. 1 was implemented in Simulink and simulated in the time domain The switch $a$ was operated at $t=0$ and the transient of the fault current $i_{\mathrm{a}}(t)$ was calculated. The source was a set of balanced voltages with positive phase rotation, phase of $e_{\mathrm{a}}(t)$ equal to $+\pi / 6$, rms amplitude $1 \mathrm{kV}$ and frequency $50 \mathrm{~Hz}$. The other parameters were selected as $R_{1}=R_{2}=R_{n 1}=R_{n 2}=0.1 \Omega, \quad L_{p}=$ $3 \mathrm{mH}, L_{m}=1 \mathrm{mH}, L_{2}=5 \mathrm{mH}$. Fig. 4a shows the comparison between $i_{\mathrm{a}}(t)$ obtained by simulation (red curve) and obtained by inverse Laplace transform of $I_{a}(s)$ as calculated in Section 3 (blue curve). In Fig. 4b the same comparison was performed by assuming the phase of source $a$ equal to $-\pi / 2$ instead of $+\pi / 6$ as in the previous case. In both cases the proposed

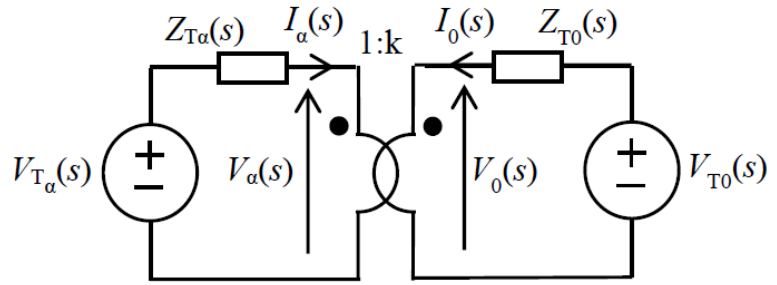

Figure 3. Coupled alpha and zero circuits in the Laplace domain for singleline-to-ground fault analysis.

analytical approach shows good agreement with simulations since the red lines are covered by the blue lines. In Fig. 5 the behaviors of $v_{\mathrm{b}}$ and $v_{\mathrm{c}}$ obtained by inverse Laplace transform of (8) are compared with simulations (source a with phase $+\pi / 6)$. Notice the discontinuous behavior at $t=0$.

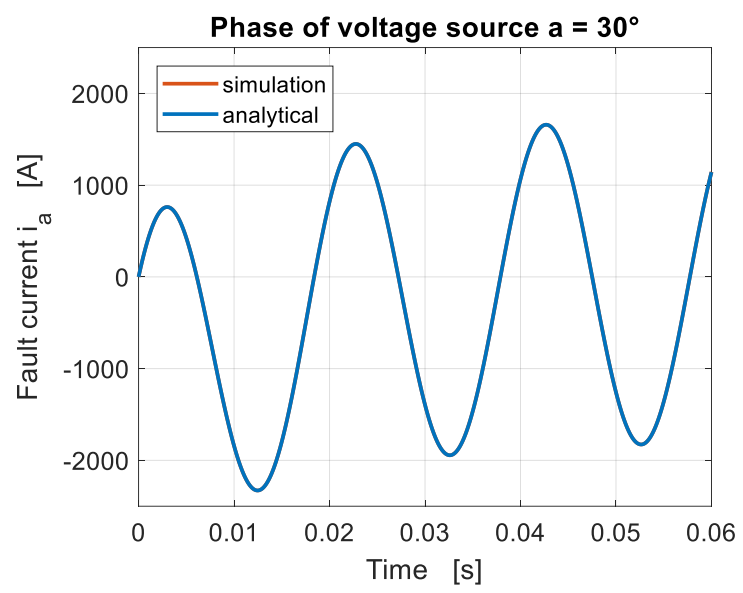

(a)

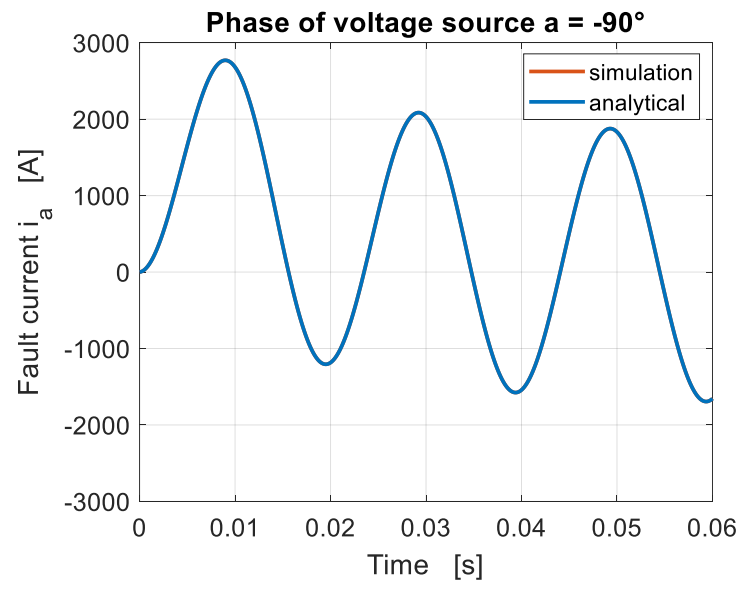

(b)

Figure 4. Comparison between numerical simulation (red curves) and analytical model (blue curves) for the transient behavior of the fault current $i_{\mathrm{a}}(t)$ in the three-phase circuit in Fig. 1. In (a) the phase of the voltage source $e_{\mathrm{a}}(t)$ was $\pi / 6$, whereas in (b) the phase was $-\pi / 2$. Red curves are exactly covered by blue curves. 


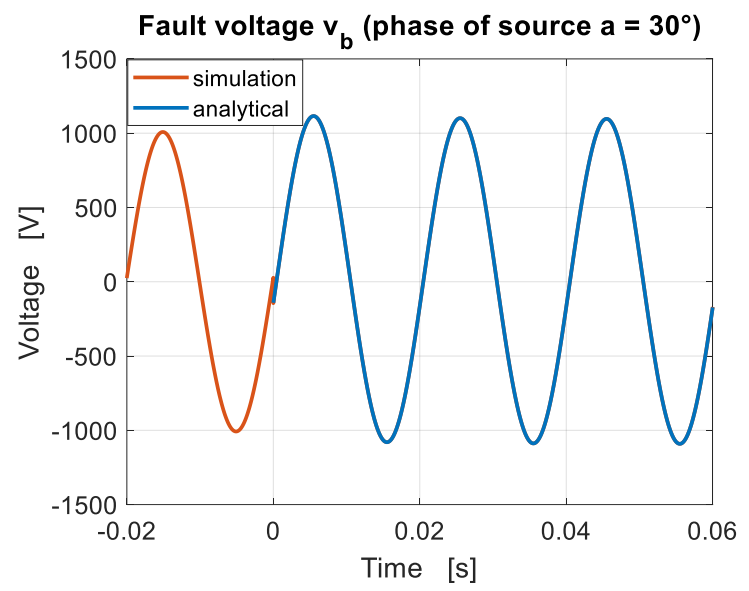

(a)

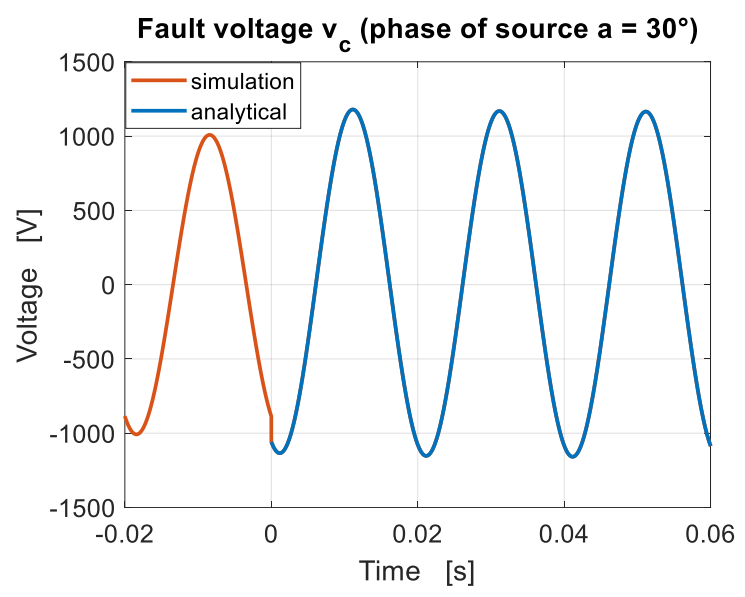

(b)

Figure 5. Comparison between numerical simulation (red curves) and analytical model (blue curves) for the transient behavior of the fault voltages $v_{\mathrm{b}}(t)$ and $v_{\mathrm{c}}(t)$ in the three-phase circuit in Fig. 1. For $t<0$ the analytical solution is not represented. For $t>0$ red curves are covered by blue curves.

\section{CONCLUSION}

It was shown that the transient analysis of single-line-toground fault can be effectively and rigorously performed by resorting to the Clarke transformation and the Thevenin equivalents in the Laplace domain. Asymmetry introduced by the fault condition results in coupling between the Clarke modal circuits. A conceptually similar result is obtained in the conventional analysis of faults under sinusoidal steady-state conditions performed by means of the symmetrical component transformation. The proposed systematic approach will be extended to other fault conditions in future work.

\section{REFERENCES}

[1] A. Greenwood, Electrical transients in power systems, 2nd ed., Wiley, 1991.

[2] M. Owen, "Transient analysis using component transforms," in Proc. of 2011 IEEE Power Engineering and Automation Conference, pp. 4-9, 2011.

[3] D. Bellan, S. A. Pignari, and G. Superti-Furga, "Consistent circuit technique for zero-sequence currents evaluation in interconnected single/three-phase power networks," Journal of Electrical Systems, vol. 12, no. 2, pp. 230-238, 2016.

[4] G. C. Paap, "Symmetrical components in the time domain and their application to power network calculations," IEEE Trans. on Power Systems, vol. 15, no. 2, pp. 522-528, 2000.

[5] D. Bellan, G. Superti-Furga, and S. A. Pignari, "Circuit representation of load and line asymmetries in three-phase power systems," International Journal of Circuits, Systems and Signal Processing, vol. 9, pp. 75-80, 2015.

[6] J. Aller, A. Bueno, and T. Paga, "Power system analysis using space vector transformation," IEEE Transactions on Power Systems, vol. 17, no. 4, pp. 957-965, 2002.

[7] G. Superti-Furga, S. Barcellona, and E. Tironi, "Space-vector approach in three-phase unbalance and distortion analysis," in Proc. of 17th International Conference on Harmonics and Quality of Power (ICHQP), 16-19 Oct. 2016, pp. 721-726.

[8] D. Bellan and G. Superti-Furga, "Space-vector state-equation analysis of three-phase transients," Journal of Electrical Systems, vol. 14, no. 1, pp. 188-198, 2018. 